\title{
IDEALS AND POWERS OF CARDINALS
}

\author{
BY KAREL PRIKRY 1
}

Communicated by Alistair Lachlan, April 28, 1975

We obtain results concerning the behaviour of the function $2^{\omega_{\alpha}} \quad(\alpha \in$ On) under the assumption of the existence of certain kind of ideals. These results complement those of Ulam [7], Tarski [6] and Solovay [4] and [5]. In particular, it follows that if $2^{\omega}$ is real-valued measurable, then $2^{\nu}=2^{\omega}$ for all infinite $\nu<2^{\omega}$.

We assume some familiarity with [4] and [5]. $\alpha, \beta, \gamma, \delta, \eta, \xi, \rho(\kappa, \lambda, \nu, \tau)$ denote ordinals (inf. cardinals). $f, g, h$ denote functions; $F$ denotes families of functions or sets. We use the Erdös-Hajnal notation $[S]^{\nu},[S]^{<\nu}$, etc. (see [2]). $F$ is $\lambda$-almost disjoint ( $\lambda$-a.d.) if $|X \cap Y|<\lambda$ whenever $X, Y \in F$ and $X \neq Y$.

Definition 1. $\kappa$ is $\lambda$-real-supercompact (abbrev. $\lambda$-r.s.c.) if there is a real-valued $\kappa$-compl. measure $\mu$ defined on $P\left([\lambda]^{<\kappa}\right)$ such that

(i) $\mu\left([\lambda]^{<\kappa}\right)=1$;

(ii) for every $\alpha \in \lambda, \mu(\{x: \alpha \notin x\})=0$;

(iii) if $\mu(X)>0$ and $f: X \rightarrow \lambda$ is such that $f(x) \in x$ for all $x \in X$, then there is $Y \subseteq X$ such that $\mu(Y)>0$ and $f$ is constant on $Y$.

$\kappa$ is r.s.c. if $\kappa$ is $\lambda$-r.s.c. for all regular $\lambda \geqslant \kappa$. We define " $\kappa$ is $\omega_{1}$-saturatedly supercompact" (abbrev. $\omega_{1}$-s.s.c.) by replacing $\mu$ by an ideal $I$ in the obvious way.

One can show by the methods of [3] and [4] that if it is consistent that a s.c. cardinal exists, then it is consistent that $2^{\omega}$ is r.s.c.

Definition 2. $R_{2}\left(\kappa_{0}, \kappa_{1}\right)$ holds if for every partition $\left[\kappa_{1}\right]^{2}=$ $\bigcup\left\{K_{\xi}: \xi \in \lambda\right\}$, where $\omega<\lambda<\kappa_{0}$, there exists an $X \subseteq \kappa_{1}$ and $M \subseteq \lambda$ such that $|X|=\kappa_{0},|M|<\lambda$, and $[X]^{2} \subseteq \bigcup\left\{K_{\xi}: \xi \in M\right\}$.

THEOREM 1. Let $\lambda, \nu<\kappa, \omega<\operatorname{cf}(\lambda)$ and $F \subseteq[\nu] \geqslant \lambda$ be $\lambda$-a.d. If $R_{2}(\kappa, \kappa)$ holds and $\operatorname{cf}(\kappa)>\omega$, then $|F|<\kappa$. If $R_{2}\left(\kappa, \kappa_{1}\right)$ holds and $\kappa_{1}$ is regular, then $|F|<\kappa_{1}$.

THEOREM 2. Set $2^{\omega}=\kappa$ and suppose that $\kappa$ carries a $\kappa$-compl. $\omega_{1}$-sat. nontrivial ideal. Then

(a) for all $\nu<\kappa, 2^{\nu}=\kappa$;

(b) if $I \subseteq P(\kappa)$ is $\omega_{1}$-compl., $\omega_{1}$-sat. and $[\kappa]<\kappa \subseteq I$, then $|P(\kappa) / I|=2^{\kappa}$;

(c) if $\nu<\kappa$ and $\operatorname{cf}(\nu)>\omega$, then there is a family $F \subseteq{ }^{\nu} \nu$ such that $|F|<\kappa$ and each $g \in{ }^{\nu} \nu$ is dominated everywhere by some $f \in F$;

\footnotetext{
AMS (MOS) subject classifications (1970). Primary $02 \mathrm{~K} 35$.

${ }^{1}$ Research supported by NSF grant GP 43841 and by a Fellowship from the Institute for Advanced Study.
} 
(d) if $\lambda, \nu<\kappa, \omega<\operatorname{cf}(\lambda)$ and $F \subseteq[\nu]^{\geqslant \lambda}$ is $\lambda$-a.d., then $|F|<\kappa$.

THEOREM 3. Suppose that $2^{\omega}=\kappa$ is $\omega_{1}$-s.s.c. Then

(a) $\lambda^{k}=\lambda$ for all regular $\lambda>\kappa$;

(b) $2^{\nu}=\nu^{+}$for all singular strong limit $\nu>\kappa$;

(c) if $I \subseteq P(\kappa)$ is $\omega_{1}$-compl., $\omega_{1}$-sat., $[\kappa]^{<\kappa} \subseteq I$ and $P(\kappa) / I$ can be generated (by infinitary Boolean operations) from $\lambda$ elements, then either $2^{\kappa}=\lambda$, or $2^{\kappa}=\lambda^{+}$and $\operatorname{cf}(\lambda)=\omega$;

(d) if $\lambda \geqslant \kappa$, then $\square_{\lambda}$ is false (see [5] for the statement of $\square_{\lambda}$ ).

Solovay [4, Lemma 14, p. 406] proved that $R_{2}(\kappa, \kappa)$ holds if $\kappa$ carries a $\kappa$-compl. $\omega_{1}$-sat. nontrivial ideal. The proof of Theorem 2(a) uses this result, Theorem 1, and Tarski's "almost disjoint sets" construction. It proceeds by induction on $\nu<2^{\omega}$.

Theorem 2(b) strengthens a result of Kunen who showed that $|P(\kappa) / I| \geqslant$ $\kappa^{+}$. To prove this, he used the fact that in the Boolean-valued universe $V^{P(\kappa) / I}$, $|P(\omega)| \geqslant \kappa^{+}$. Theorem 2(a) enables us to show that in $V^{P(\kappa) / I},|P(\omega)|=2^{\kappa}$.

To prove Theorem 2(c), we again use a method of Kunen who showed that the corresponding result holds for ${ }^{\omega} \omega$ if $2^{\omega}$ is r.v.m. This is made possible by Theorem 2(a). The method involves considering Solovay's Boolean ultrapower $V^{\kappa} / I$.

The proof of Theorem 3 involves ideas of $[5, \S \S 3$ and 4$]$ and an additional unpublished result of Solovay.

Lemma 1 (Solovay, UNPUBliShed). For every regular $\lambda>\omega$ there exists an $\omega$-ary Jónsson algebra $\langle\lambda, f\rangle$ such that for every $X \subseteq \lambda,\left|\operatorname{mg}\left(f \backslash[X]^{\omega}\right)\right|$ $\leqslant|X|$.

Lemma 2. Let $\lambda \geqslant \kappa$ be regular and $\mu$ be a measure as in Definition 1 .

(a) If $X \subseteq[\lambda]^{<\kappa}$ and $\mu(X)=1$, then $|X|=\lambda^{k}$.

(b) Let $g:[\lambda]^{<\kappa} \rightarrow \lambda$ be defined by $g(x)=\sup (x)$. Then there is $X \subseteq$ $[\lambda]^{<\kappa}$ such that $\mu(X)=1$ and $g \nmid X$ is one-to-one.

The proof of Lemma 2(a) uses Theorem 2(a). Lemma 2(b) is analogous to Theorem 2 of [5]. The proof of Lemma 2(b) uses Lemma 1 where Solovay's proof of his Theorem 2 used an older result of [1]. Some modifications are required and this holds for the proof of Theorem 3(d) as well. Theorem 3(a) follows from Lemma 2 and implies Theorem 3(b). Theorem 3(c) follows from Theorem 2(b) and Theorem 3(a).

The author wishes to thank $\mathrm{K}$. Kunen for valuable discussions concerning the subject of this note.

\section{REFERENCES}

1. P. Erdös and A. Hajnal, On a problem of B. Jónsson, Bull. Acad. Polon. Sci. Sér. Sci. Math. Astronom. Phys. 14 (1966), 19-23. MR 35 \#64. 
2. P. Erdös and A. Hajnal, Unsolved problems in set theory, Proc. Sympos. Pure Math., vol. 13, part 1, Amer. Math. Soc., Providence, R. I., 1971, pp. 17-48. MR 43 \#6101.

3. K. McAloon, Consistency results about ordinal definability, Ann. Math. Logic 2 (1970/71), no. 4, 449-467. MR 45 \#1753.

4. R. Solovay, Real-valued measurable cardinals, Proc. Sympos. Pure Math., vol. 13, part 1, Amer. Math. Soc., Providence, R. I., 1971, pp. 397-428. MR 45 \#55.

5. - Strongly compact cardinals and the GCH, Proc. Sympos. Pure Math., vol. 25, Amer. Math. Soc., Providence, R. I., 1974, pp. 365-372.

6. A. Tarski, Ideale in vollständigen Mengenkörpern. II, Fund. Math. 33 (1945), 51 65. MR 8, 193.

7. S. Ulam, Zur Masstheorie in der algemeinen Mengenlehre, Fund. Math. 16 (1930), 140-150.

DEPARTMENT OF MATHEMATICS, UNIVERSITY OF MINNESOTA, MINNEAPOLIS, MINNESOTA 55455 